UNIL-IPT-00-28

December 2000

\title{
A Warped Supersymmetric Standard Model
}

\author{
Tony Gherghetta ${ }^{a}$ and Alex Pomarol ${ }^{b}$ \\ ${ }^{a}$ IPT, University of Lausanne, CH-1015 Lausanne, Switzerland \\ ${ }^{b}$ IFAE, Universitat Autònoma de Barcelona, E-08193 Barcelona, Spain
}

\begin{abstract}
We study the breaking of supersymmetry in five-dimensional (5d) warped spaces, using the Randall-Sundrum model as a prototype. In particular, we present a supersymmetrybreaking mechanism which has a geometrical origin, and consists of imposing different boundary conditions between the fermions and bosons living in the $5 \mathrm{~d}$ bulk. The scale of supersymmetry breaking is exponentially small due to the warp factor of the AdS metric. We apply this mechanism to a supersymmetric standard model where supersymmetry breaking is transmitted through the AdS bulk to matter fields confined on the Planckbrane. This leads to a predictable superparticle mass spectrum where the gravitino mass is $10^{-3} \mathrm{eV}$ and scalar particles receive masses at the one-loop level via bulk gauge interactions. We calculate the mass spectrum in full detail using the $5 \mathrm{~d}$ AdS propagators. The AdS/CFT correspondence suggests that our 5d warped model is dual to the ordinary $4 \mathrm{~d}$ MSSM with a strongly coupled CFT sector responsible for the breaking of supersymmetry.
\end{abstract}




\section{Introduction}

The standard model is believed to be an effective theory valid up to some energy scale near the electroweak scale. What lies beyond the standard model has been the subject of active research. Among the possible candidates, there are technicolor theories, supersymmetry, and, recently, extra dimensions [1].

Extra dimensions and supersymmetry present an additional motivation. They could be an important ingredient in the underlying theory that includes a quantum description of gravity, and in particular for string theory they play a crucial role. A particularly interesting extra dimension scenario is the Randall-Sundrum model [2]. In this model the extra dimension is compactified in a slice of anti-deSitter (AdS) space, and, as a consequence, the electroweak scale is generated by an exponential warp factor in the metric. This model can be supersymmetrized [3, 4, 5] providing an interesting alternative to the minimal supersymmetric standard model (MSSM), and a possible connection to string theories [6].

In this article we want to continue the study of supersymmetric extensions of the standard model living in five dimensions where the extra dimension is compactified as in the RandallSundrum model [3, 7]. In particular, we want to study supersymmetry breaking. A warped extra dimension allows for new ways of breaking supersymmetry. The particular mechanism that we will consider here consists of imposing different boundary conditions between the fermions and bosons. This supersymmetry breaking mechanism has been previously studied in flat space but not in warped spaces. In warped space this way of breaking supersymmetry leads to novel phenomenological consequences. For example, the radius of compactification does not need to be large $\left(\mathrm{TeV}^{-1}\right)$ as in the case of flat space [8]. Therefore it can be consistent with a large cut-off scale that is related with the Planck scale, $M_{P}$, or grand unified theories [9], 7]. As we will show, the scale of supersymmetry breaking can be very low $(\sim \mathrm{TeV})$ and this implies a superlight gravitino $m_{3 / 2} \sim 10^{-3} \mathrm{eV}$. Scalars are massless at tree-level and get masses at the one-loop level. We will study in detail a "warped" version of the MSSM, where gravity and gauge bosons live in the five-dimensional (5d) AdS bulk, while matter fields are located on one of the boundaries, the Planck-brane. In this warped MSSM the squark and slepton masses arise at one-loop from the gauge interactions and are therefore naturally flavor independent. One of the most interesting properties of the model is its predictivity of the low-energy mass spectrum. We will calculate it here in full detail. Although we present the calculation for a particular extension of the standard model, the calculation of quantum effects in warped spaces that we present here is much more general and can also be useful for other scenarios.

Another important motivation for the study of the MSSM in a slice of AdS arises from the AdS/CFT correspondence [10]. This conjecture suggests that these 5 d models have a stronglycoupled $4 \mathrm{~d}$ dual [11, 12, 13, 14]. Therefore, the study of the weakly coupled $5 \mathrm{~d}$ gravity theory here will be helpful in understanding supersymmetric 4d theories with a strongly coupled sector. We will comment later on this duality.

In section 2 we introduce the Randall-Sundrum compactification and its supersymmetric version. In particular we also analyze the gravitino Kaluza-Klein decomposition since it is the 
only field not considered in Ref. [3] (see also [15]). In section 3, we present the supersymmetrybreaking mechanism, which is based on imposing "twisted" boundary conditions for fermions in the bulk, and comment on the differences compared with the case of a flat extra dimension. In section 4 we introduce a version of the MSSM living in a slice of AdS and calculate the sparticle mass spectrum, at tree-level and at one-loop level. We will also comment on the holographic interpretation of the model. Our concluding remarks appear in Section 5. Finally in the Appendix, we present a detailed calculation of the $5 \mathrm{~d}$ propagators in a slice of AdS.

\section{The Warped Supersymmetric Brane-World}

We will consider the scenario of Ref. [2], which is based on a nonfactorizable 5d geometry. The fifth dimension $y$ is compactified on an orbifold, $S^{1} / Z_{2}$ of radius $R$, with $0 \leq y \leq \pi R$. The boundary of the $5 \mathrm{~d}$ spacetime consists of two 3-branes located at the orbifold fixed points $y^{*}=0$ and $y^{*}=\pi R$. This configuration with the $5 \mathrm{~d}$ metric solution [2]

$$
d s^{2}=e^{-2 \sigma} \eta_{\mu \nu} d x^{\mu} d x^{\nu}+d y^{2} \equiv g_{M N} d x^{M} d x^{N}
$$

is a slice of AdS space, where $\sigma=k|y|$ and $1 / k$ is the AdS curvature radius. The 5 d coordinates are labelled by capital Latin letters, $M=(\mu, 5)$ where $\mu=0, \ldots, 3$. The complete supergravity action for this configuration is obtained by including the gravitino and graviphoton together

with the graviton [3, 4, 5]. However, for the discussion of supersymmetry breaking it will suffice to only consider the additional gravitino kinetic and mass terms, which are given by [3]

$$
\begin{aligned}
S & =S_{5}+S_{(0)}+S_{(\pi R)}, \\
S_{5} & =\int d^{4} x \int d y \sqrt{-g}\left[-\frac{1}{2} M_{5}^{3}\left(\mathcal{R}+i \bar{\Psi}_{M}^{i} \gamma^{M N P} D_{N} \Psi_{P}^{i}-i \frac{3}{2} \sigma^{\prime} \bar{\Psi}_{M}^{i} \gamma^{M N}\left(\sigma_{3}\right)^{i j} \Psi_{N}^{j}\right)-\Lambda\right], \\
S_{\left(y^{*}\right)} & =\int d^{4} x \sqrt{-g_{4}}\left[\mathcal{L}_{\left(y^{*}\right)}-\Lambda_{\left(y^{*}\right)}\right],
\end{aligned}
$$

where $g_{4}$ is the induced metric on the 3-brane located at $y^{*}$, and $\gamma^{M_{1} M_{2} \ldots M_{n}}=\frac{1}{n !} \gamma^{\left[M_{1}\right.} \gamma^{M_{2}} \ldots \gamma^{\left.M_{n}\right]}$ is the antisymmetrized product of gamma matrices. We have defined $\sigma^{\prime}=d \sigma / d y$. Supersymmetry automatically ensures the bulk/boundary conditions $\Lambda_{(0)}=-\Lambda_{(\pi R)}=-\Lambda / k$. The action contains the $5 \mathrm{~d}$ Planck scale $M_{5}$, the $5 \mathrm{~d}$ Ricci scalar $\mathcal{R}$, two symplectic-Majorana gravitinos, $\Psi_{M}^{i}(i=1,2)$, and a bulk cosmological constant $\Lambda$. At $y^{*}=0$ the effective $4 \mathrm{~d}$ mass scale is of order of the Planck scale, $M_{P}^{2} \simeq M_{5}^{3} / k$, and we will refer to the brane there as the Planck-brane. Similarly, at $y^{*}=\pi R$ the effective mass scale is of order $M_{P} e^{-\pi k R}$, which is the TeV scale for $k R \simeq 11$. Consequently the 3 -brane located there will be referred to as the TeV-brane. The index $i$ labels the fundamental representation of the $\mathrm{SU}(2)_{R}$ automorphism group of the $N=1$ supersymmetry algebra in five dimensions. The gravitino supersymmetry transformation is given by [3]

$$
\delta \Psi_{M}^{i}=D_{M} \eta^{i}+\frac{\sigma^{\prime}}{2} \gamma_{M}\left(\sigma_{3}\right)^{i j} \eta^{j}
$$


where the symplectic-Majorana spinor $\eta^{i}$ is the 5 d supersymmetry parameter.

Similarly, gauge bosons and matter can be added to the bulk [16, 17, 9, 18, 3]. In a $5 \mathrm{~d}$ supersymmetric theory they form part of vector supermultiplets and hypermultiplets. The behavior of these supermultiplets in the background of Eq. (1) was considered in Ref. [3], where the Kaluza-Klein mass spectrum was also derived. Only the analysis of the gravitino field was not presented in Ref. [3]. For this reason, we will present below the Kaluza-Klein decomposition of the gravitino. This will also help to show how the superHiggs mechanism operates levelby-level in the Kaluza-Klein modes, and will help to better understand the supersymmetry breaking mechanism presented in the next section.

\subsection{Kaluza-Klein decomposition of the gravitino and the superHiggs mechanism level by level}

Let us start by decomposing the 5 d gravitino, $\Psi_{M}$, and the 5 d supersymmetry parameter, $\eta$, into 4d Kaluza-Klein fields

$$
\begin{aligned}
\Psi_{\mu L, R}\left(x^{\mu}, y\right) & =\sum_{n=0}^{\infty} \psi_{\mu L, R}^{(n)}\left(x^{\mu}\right) f_{L, R}^{(n)}(y) \\
\Psi_{5 L, R}\left(x^{\mu}, y\right) & =\sum_{n=0}^{\infty} \psi_{5 L, R}^{(n)}\left(x^{\mu}\right) f_{5 L, R}^{(n)}(y) \\
\eta_{L, R}\left(x^{\mu}, y\right) & =\sum_{n=0}^{\infty} \eta_{L, R}^{(n)}\left(x^{\mu}\right) f_{L, R}^{(n)}(y) .
\end{aligned}
$$

We have dropped the $\mathrm{SU}(2)_{R}$ index $i$, since we need only consider the $i=1$ component (the $i=2$ component is simply obtained from the symplectic-Majorana condition). We have also defined $\gamma_{5} \Psi_{L, R}= \pm \Psi_{L, R}$. It is important to note that we have chosen the $y$-dependent wavefunction of the supersymmetry parameter $\eta$ to be the same as that for the Kaluza-Klein gravitinos.

\subsubsection{Kaluza-Klein modes $n \neq 0$}

The supersymmetry transformation Eq. (3) for $i=1$ gives

$$
\begin{aligned}
\delta \Psi_{\mu} & =\partial_{\mu} \eta+\sigma^{\prime} \gamma_{\mu}\left(\frac{1-\gamma_{5}}{2}\right) \eta, \\
\delta \Psi_{5} & =\partial_{5} \eta+\sigma^{\prime} \frac{\gamma_{5}}{2} \eta .
\end{aligned}
$$


Substituting Eq. (4) into Eq. (5) and projecting out the $n$ th-mode \& we find that the supersymmetry transformation for the $n$th Kaluza-Klein gravitino mode is given by

$$
\begin{aligned}
\delta \psi_{\mu L}^{(n)} & =\partial_{\mu} \eta_{L}^{(n)}+\tilde{\gamma}_{\mu} \sum_{k=0}^{\infty} a_{n k} \eta_{R}^{(k)}, \\
\delta \psi_{\mu R}^{(n)} & =\partial_{\mu} \eta_{R}^{(n)},
\end{aligned}
$$

where $\tilde{\gamma}_{\mu}$ is the $4 \mathrm{~d}$ Minkowski gamma matrix and the coefficients $a_{n k}$ are given by

$$
a_{n k} \equiv \int d y e^{-2 \sigma} \sigma^{\prime} f_{L}^{(n)}(y) f_{R}^{(k)}(y)
$$

The coefficients $a_{n k}$ imply that the supersymmetry transformation of $\psi_{\mu L}^{(n)}$ at level $n$, depends nontrivially on the complete tower of Kaluza-Klein parameters $\eta_{R}^{(k)}$. This effect is entirely due to the fact that the bulk is AdS. Let us now impose the following relation for the wavefunctions of $\Psi_{5}$ :

$$
f_{5 L, R}^{(n)}=\frac{1}{m_{n}}\left( \pm \partial_{5}+\frac{1}{2} \sigma^{\prime}\right) f_{L, R}^{(n)},
$$

where $m_{n}$ is the $4 \mathrm{~d}$ mass of the gravitino Kaluza-Klein mode $n$, which will be derived below. The condition (9) allows us to write a simple expression for the supersymmetry transformation of Kaluza-Klein modes $\psi_{5}^{(n)}$

$$
\begin{aligned}
\delta \psi_{5 L}^{(n)} & =m_{n} \eta_{L}^{(n)}, \\
\delta \psi_{5 R}^{(n)} & =-m_{n} \eta_{R}^{(n)} .
\end{aligned}
$$

This shows that the $n$th Kaluza-Klein mode of the 5 th component of the gravitino transforms as a Goldstino of the $\eta^{(n)}$ supersymmetry transformation and that these $N=2$ supersymmetries are non-linearly realized. We can now see that the redefined gravitinos

$$
\begin{aligned}
\widetilde{\psi}_{\mu L}^{(n)} & \equiv m_{n} \psi_{\mu L}^{(n)}-\partial_{\mu} \psi_{5 L}^{(n)}+m_{n} \tilde{\gamma}_{\mu} \sum_{k=0}^{\infty} a_{n k} \frac{\psi_{5 R}^{(k)}}{m_{k}}, \\
\widetilde{\psi}_{\mu R}^{(n)} & \equiv m_{n} \psi_{\mu R}^{(n)}+\partial_{\mu} \psi_{5 R}^{(n)},
\end{aligned}
$$

are invariant under supersymmetry transformations, and therefore correspond to the physical fields. On the contrary, the fields $\psi_{5}^{(n)}$ are gauge dependent and can be eliminated. This is the superHiggs mechanism. The $\psi_{5}^{(n)}$ are eaten by the gravitino $\psi_{\mu}^{(n)}$ to become massive.

Let us now turn to the Rarita-Schwinger equation for the bulk gravitino, which in the AdS background reads

$$
\gamma^{M N P} D_{N} \Psi_{P}-\frac{3}{2} \sigma^{\prime} \gamma^{M P} \Psi_{P}=0
$$

1 This corresponds to multiplying each side of Eq. (5) by $f_{L, R}^{(n)}$, integrating over $y$, and using the gravitino orthogonality condition $\int d y e^{-\sigma} f_{L, R}^{(n)} f_{L, R}^{(m)}=\delta_{n m}$. 
Using the redefined gravitino fields (12), the equation of motion (13) simplifies to

$$
\gamma^{\mu \nu \rho} \partial_{\nu} \widetilde{\psi}_{\rho L, R}^{(n)}-m_{n} \gamma^{\mu \rho} \widetilde{\psi}_{\rho R, L}^{(n)}=0,
$$

which represents the $4 \mathrm{~d}$ massive Rarita-Schwinger equation for the spin $3 / 2$ field $\widetilde{\psi}_{\mu}^{(n)}$, and where the $y$-dependent Kaluza-Klein wavefunctions satisfy

$$
\begin{aligned}
& \left(\partial_{5}+\frac{1}{2} \sigma^{\prime}\right) f_{L}^{(n)}=m_{n} e^{\sigma} f_{R}^{(n)}, \\
& \left(\partial_{5}-\frac{5}{2} \sigma^{\prime}\right) f_{R}^{(n)}=-m_{n} e^{\sigma} f_{L}^{(n)} .
\end{aligned}
$$

One can see that the dependence on $\psi_{5}^{(n)}$ has dropped out and the equation of motion depends, as expected, only on $\widetilde{\psi}_{\mu}^{(n)}$. The solutions of Eqs. (15) and (16) are a special case of the general solution appearing in Ref. [3]. In fact, defining $\widehat{f}_{L, R}^{(n)}=e^{-\sigma} f_{L, R}^{(n)}$ one can see that $\widehat{f}_{L, R}^{(n)}$ corresponds to the wavefunction of a "hatted" fermion of mass $m=3 \sigma^{\prime} / 2$ defined in Ref. [3]. Thus, using the results in Ref. [3] and the fact that $\Psi_{\mu L}\left(\Psi_{\mu R}\right)$ are defined even (odd) under the $Z_{2}$-parity, we obtain the $y$-dependent gravitino wavefunctions

$$
\begin{aligned}
f_{L}^{(n)} & =\frac{1}{N_{n}} e^{\frac{3}{2} \sigma}\left[J_{2}\left(\frac{m_{n}}{k} e^{\sigma}\right)+b\left(m_{n}\right) Y_{2}\left(\frac{m_{n}}{k} e^{\sigma}\right)\right], \\
f_{R}^{(n)} & =\frac{\sigma^{\prime}}{k N_{n}} e^{\frac{3}{2} \sigma}\left[J_{1}\left(\frac{m_{n}}{k} e^{\sigma}\right)+b\left(m_{n}\right) Y_{1}\left(\frac{m_{n}}{k} e^{\sigma}\right)\right],
\end{aligned}
$$

where $J_{\alpha}$ and $Y_{\alpha}$ are Bessel functions, $N_{n}$ are normalization constants and the coefficients $b\left(m_{n}\right)$ satisfy

$$
\begin{aligned}
& b\left(m_{n}\right)=-\frac{J_{1}\left(\frac{m_{n}}{k}\right)}{Y_{1}\left(\frac{m_{n}}{k}\right)}, \\
& b\left(m_{n}\right)=b\left(m_{n} e^{\pi k R}\right) .
\end{aligned}
$$

The Kaluza-Klein masses of the gravitinos $\widetilde{\psi}_{\mu}^{(n)}$ can be obtained by solving (20), and for $n>0$ they are approximately given by

$$
m_{n} \simeq\left(n+\frac{1}{4}\right) \pi k e^{-\pi k R} .
$$

Finally, using (15) and (16), and the fact that $\Psi_{5 R}\left(\Psi_{5 L}\right)$ are even (odd) under the $Z_{2}$-parity, we have from the condition (9)

$$
\begin{aligned}
f_{5 L}^{(n)} & =e^{\sigma} f_{R}^{(n)} \\
f_{5 R}^{(n)} & =e^{\sigma} f_{L}^{(n)}-\frac{2 \sigma^{\prime}}{m_{n}} f_{R}^{(n)} .
\end{aligned}
$$




\subsubsection{Massless sector}

The $y$-dependence of the gravitino zero-mode wavefunction is obtained from Eq. (15), since under the orbifold symmetry, $f_{R}^{(0)}$ is projected out. Thus for the remaining mode, $f_{L}^{(0)}$ with $m_{0}=0$, we obtain

$$
f_{L}^{(0)}(y)=\frac{1}{\sqrt{N}_{0}} e^{-\frac{1}{2} \sigma}
$$

where the normalization factor $N_{0}=\left(1-e^{-2 \pi k R}\right) / k$. This is consistent with the $y$-dependence of the graviton zero-mode wavefunction, as expected from supersymmetry. Similarly, $\eta_{R}^{(0)}$ is projected out and $\eta_{L}^{(0)}$ whose wavefunction is also given by Eq. (24) parametrizes the remaining $N=1$ supersymmetry of the theory. In fact one can check that Eq. (24) satisfies the Killing spinor condition.

Similarly, for the fifth-component of the gravitino, we have that $\psi_{5 L}^{(0)}$ is projected out and only $\psi_{5 R}^{(0)}$ remains in the theory. This corresponds to the supersymmetric partner of the radion, the "radino". The 4d effective Lagrangian of this field has been recently presented in Ref. [19].

\section{Supersymmetry breaking in a slice of AdS}

Different mechanisms of supersymmetry breaking in brane-world scenarios have been considered in the past. The most popular, based on the Horava-Witten model, corresponds to breaking supersymmetry in a hidden-sector living on a brane located at a finite distance from the observable-sector brane [20]. The moduli (e.g. the dilaton and radion) play the role of messengers communicating the supersymmetry-breaking from the hidden to the observable sector. These scenarios rely on gaugino condensation to occur on the hidden-sector brane in order to explain the hierarchy.

Warped (AdS) spaces allow for new possibilities. First of all, since the hierarchy is now explained by the warp factor, one does not need a gaugino condensation in a hidden sector to be responsible for a small supersymmetry breaking. Supersymmetry can be broken at tree-level if it occurs on the TeV-brane, and therefore have a stringy origin.

The supersymmetry-breaking mechanism that we will consider here is based on imposing different boundary conditions between fermions and bosons on the TeV-brane. This breaks supersymmetry for the bulk fields, and as we shall see, the Kaluza-Klein fermions and bosons receive $\mathrm{TeV}$ mass-splittings. The mechanism consists of the following. The $5 \mathrm{~d}$ bulk fields in the supersymmetric $Z_{2}$ orbifold can be classified as either odd or even fields under the $Z_{2}$ parity. For the $5 \mathrm{~d}$ fermions, we have two possibilities to define the $Z_{2}$ parity, namely

$$
\psi(-y)= \pm \gamma_{5} \psi(y)
$$

Once a choice is made, this also defines the chirality on the $4 \mathrm{~d}$ boundary at $y^{*}=0$, since $\psi(0)= \pm \gamma_{5} \psi(0)$. For the supersymmetric $Z_{2}$ orbifold the same chirality is chosen on the two 
boundaries at $y^{*}=0$ and $y^{*}=\pi R$. In this way only half of the bulk supersymmetry is broken by the boundaries, leaving an $N=1$ supersymmetric theory at the massless level. However, there also exists the possibility to separately define the chirality of fermions on the two boundaries. For example, the choice

$$
\begin{aligned}
\psi(0) & =\gamma_{5} \psi(0), \\
\psi(\pi R) & =-\gamma_{5} \psi(\pi R),
\end{aligned}
$$

corresponds to the following $y$-dependence

$$
\begin{aligned}
\psi(-y) & =\gamma_{5} \psi(y), \\
\psi(-y+\pi R) & =-\gamma_{5} \psi(y+\pi R) .
\end{aligned}
$$

Thus, under a $2 \pi$ rotation around the circle $S^{1}$, Eq. (27) leads to fermions that are antiperiodic

$$
\psi(y+2 \pi R)=-\psi(y) .
$$

As will be shown below, the boundary conditions (26) project out the massless fermion modes arising from bulk fields. Also supersymmetry is now completely broken since no Killing spinor can be defined 2 .

The fermionic boundary conditions (26) have been considered previously in the literature. If the space of the extra dimension is flat, imposing these boundary conditions exactly corresponds to breaking supersymmetry by the Scherk-Schwarz mechanism [22]. This mechanism has been applied to the MSSM in Refs. [8, 23, 24, 25, 26]. In the Horava-Witten theory this was studied in Ref. [27]. However, for warped spaces the fermionic boundary conditions Eq. (26) do not correspond to the Scherk-Schwarz mechanism, because this requires a smooth limit where supersymmetry is restored [22]. In the case of warped spaces, we have not found such a smooth limit 3 .

Let us now study the fermionic spectrum with the boundary conditions (26), which we will refer to as "twisted" boundary conditions. The resulting Kaluza-Klein mass spectrum for $\psi_{L}$ is now determined by (see Appendix)

$$
\frac{J_{\alpha-1}\left(\frac{m_{n}}{k}\right)}{Y_{\alpha-1}\left(\frac{m_{n}}{k}\right)}=\frac{J_{\alpha}\left(\frac{m_{n}}{k} e^{\pi k R}\right)}{Y_{\alpha}\left(\frac{m_{n}}{k} e^{\pi k R}\right)},
$$

where $\alpha=|c+1 / 2|$ for a fermion of Dirac-type mass $m=c \sigma^{\prime}$ and $\alpha=2$ for the gravitino. One can easily check that the equation resulting from imposing twisted boundary conditions on $\psi_{R}$ leads to an identical Kaluza-Klein spectrum. The first thing to notice in Eq. (29) is that $m_{n}=0$ is no longer a solution of the above equation and therefore no massless fermions are

\footnotetext{
${ }^{2}$ This has some similarities with finite temperature which also breaks supersymmetry [21].

${ }^{3}$ Similarly, one can also show that the models of Refs. [24, 25] with only one Higgs hypermultiplet (instead of two) do not have this smooth limit to a supersymmetric theory. This alternative has recently been considered in Ref. [26], where it was shown that the boundary conditions Eq. (26) can also be understood as compactifying on a $S^{1} /\left(Z_{2} \times Z_{2}\right)$ orbifold.
} 
present. Consequently, supersymmetry is now completely broken. One can also see that the Killing spinor, $\eta_{L}^{(0)}$, whose wavefunction is identical to Eq. (24), is not consistent with the new boundary conditions. Thus, the only change with respect to Section 2.1 is that Eq. (20) is now replaced by Eq. (29), and the massless sector of subsection 2.1.2, is no longer present in the theory. Notice that from Eq. (10) the Goldstino of the broken $N=1$ supersymmetry is $\psi_{5 L}^{(0)}$.

In the limit $m_{n} \ll k$ and $k R \gg 1$, the solution of Eq. (29) is given by

$$
m_{n} \simeq\left(n+\frac{\alpha}{2}-\frac{1}{4}\right) \pi k e^{-\pi k R} .
$$

Comparing with the result for "untwisted" boundary conditions [3], one finds that the KaluzaKlein mass spectrum is shifted by a value that asymptotically approaches $1 / 2\left(\pi k e^{-\pi k R}\right)$. This is to be contrasted with the flat case where the shift in the Kaluza-Klein mass spectrum is $1 /(2 R)$.

There is an important difference when this type of supersymmetry breaking is realized in warped spaces compared to the flat case. In flat spaces this type of supersymmetry breaking is global. To see this, let us consider an observer living on one of the branes with the other brane sent to infinity $(R \rightarrow \infty)$. In this limit and in flat space, supersymmetry is restored because the Kaluza-Klein spectrum becomes continuous (the scalar-fermion mass splitting disappears). This is related to the fact that one can locally (i.e., on either brane) define a supersymmetric theory. Breaking supersymmetry globally (when the extra dimension is compact, no Killing spinor can be defined in the whole space) leads to the important property that the vacuum energy and the one-loop scalar masses are finite and independent of the cut-off scale [28, 25].

In warped spaces the situation is different and the finiteness of the one-loop scalar masses depends on which particular brane the observable sector lives. Consider first the observable sector on the Planck-brane where the TeV-brane is sent off to infinity. In this limit supersymmetry is restored on the Planck-brane because the Kaluza-Klein spectrum becomes continuous. Therefore the one-loop scalar masses on the Planck-brane will be finite. Alternatively, suppose that the observable sector is on the TeV-brane. Now, even if we consider the limit where we send the Planck brane away $(R \rightarrow \infty)$, the Kaluza-Klein spectrum remains discrete and supersymmetry stays broken. Therefore on the TeV-brane supersymmetry is broken and corrections to scalar masses will be sensitive to the ultraviolet cut-off. Another way to see that supersymmetry is broken by the TeV-brane (contrary to the flat case) is that no Killing spinor can be defined if fermions have twisted boundary conditions. Even in a non-compact space, the TeV-boundary breaks all the supersymmetries. These expectations will be confirmed in the following sections by the explicit calculation of the one-loop scalar masses in a warped AdS space.

Finally, as an alternative to the supersymmetry-breaking mechanism considered above, there also exists the possibility of breaking supersymmetry by the $F$-term, $F_{T}$ of the radion field $T$. This can easily be achieved by turning on a constant term, $W$, in the superpotential localized on the TeV-brane. In flat space this is known to generate a vacuum expectation value (VEV) $\left\langle F_{T}\right\rangle \sim W / M_{5}^{3}$. In fact in flat space, this corresponds exactly to the Scherk-Schwarz mechanism 
[29] or to imposing the twisted boundary condition for the fermion as in Eq. (26). However, in a warped space this is not the case, and a nonzero $\left\langle F_{T}\right\rangle$ leads to a new way of breaking supersymmetry. Furthermore, in a warped space the VEV of $F_{T}$, induced by a constant term in the superpotential at the TeV-brane, is exponentially suppressed, $\left\langle F_{T}\right\rangle \sim e^{-\pi k R} W / M_{5}^{3}$. The tree-level spectrum is easily derived. For the gaugino we have $m_{\lambda} \sim\left\langle F_{T} / T\right\rangle \sim \mathrm{TeV}$, while for scalars localized on either brane their masses are zero. The scalar masses are, however, induced at the one-loop level. This scenario leads, qualitatively, to the same mass spectrum as the one considered above, and will not be pursued here.

\section{The Warped MSSM}

Let us now present a candidate MSSM based on the 5d model described above. We will assume that both gravity and gauge fields are in the bulk. Supersymmetry is spontaneously broken by imposing twisted boundary conditions, (26), on the gravitino and gaugino. The MSSM matter fields are assumed to be completely localized on the Planck brane. At tree level, the matter fields are massless and the dominant supersymmetry-breaking effects will be transmitted to the matter fields on the Planck brane by the $5 \mathrm{~d}$ gauge interactions. Thus, the soft masses on the Planck brane will arise via radiative corrections and can be computed using the $5 \mathrm{~d}$ AdS propagators. On the other hand, the Higgs field can also be assumed to be a bulk field. However, in this case we will see that radiative corrections to the Higgs soft masses are sensitive to TeV-scale physics. Other alternative scenarios will also be discussed. Since the bulk fields live in a warped space, these models will be referred to as the "warped MSSM".

Before proceeding to calculate the sparticle spectrum an important comment is in order. Since the gauge bosons live in a warped extra dimension, the effective $4 \mathrm{~d}$ coupling is given by $g^{2}=\left(g_{5}^{2} k\right) /(\pi k R)$, where $g_{5}$ is the 5 d gauge coupling [17, 9]. In order to explain the Planck-TeV scale hierarchy we need $k R \simeq 10$, which implies that for $g_{5}^{2} k \lesssim 1$, we obtain $g^{2} \lesssim 1 / 30$. This is in contradiction with the experimental values of the gauge couplings which require $g^{2} \sim \mathcal{O}(1)$. Therefore, in order to agree with the experimental values one requires that $g_{5}^{2} k \gtrsim 30$. This inevitably means that the theory is close to the strong coupling regime at energies $E \sim k$. On the TeV-brane this corresponds to energies $E \sim k e^{-\pi k R}$. At these energies the expansion parameter becomes $g_{5}^{2} k /\left(16 \pi^{2}\right) \sim 0.2$. We will assume that the effects from the strong coupling regime do not spoil the AdS geometry. Similarly, we will be able to trust our low-energy predictions, provided that the energy of the processes satisfies $E \lesssim k e^{-\pi k R}$.

\subsection{Tree-level masses}

If twisted boundary conditions are imposed on the the fermions in the bulk, then all the $4 \mathrm{~d}$ fermion modes will receive masses. In particular, the zero mode of the gravitino will receive a mass whose magnitude can easily be obtained by solving (29) for $\alpha=2$ :

$$
m_{3 / 2} \simeq \sqrt{8} k e^{-2 \pi k R} .
$$


Thus, for $k=M_{P}$ and $k e^{-\pi k R}=\mathrm{TeV}$ we obtain $m_{3 / 2} \simeq 2.8 \times 10^{-3} \mathrm{eV}$. This is a superlight gravitino, as compared to the usual gravity-mediated and gauge-mediated scenarios in four dimensions, and satisfies the usual experimental constraints from cosmology and collider experiments [30. In the warped case the small gravitino mass arises because the coupling of the gravitino to the TeV-brane is exponentially suppressed, and therefore it is very insensitive to the twisting of boundary conditions on the TeV-brane. The higher Kaluza-Klein gravitino modes are approximately given by

$$
m_{n} \simeq\left(n+\frac{3}{4}\right) \pi k e^{-\pi k R}
$$

Notice that compared to the untwisted gravitino Kaluza-Klein spectrum (21), the twisted mass spectrum has indeed shifted approximately by an amount $1 / 2\left(\pi k e^{-\pi k R}\right)$. This shift is much larger than for the zero mode, because the nonzero Kaluza-Klein gravitino modes are localized near the TeV-brane and therefore couple more strongly to the $\mathrm{TeV}$ brane as compared to the the gravitino zero mode which is localized near the Planck brane.

Similarly, the tree-level gaugino mass is obtained by solving (29) with $\alpha=1$ :

$$
m_{\lambda} \simeq \sqrt{\frac{2}{\pi k R}} k e^{-\pi k R} .
$$

Thus, for $k=M_{P}$ and $k e^{-\pi k R}=\mathrm{TeV}$ we obtain $m_{\lambda} \simeq 0.24 \mathrm{TeV}$. Notice that unlike the gravitino zero mode, the gaugino zero mode receives a TeV-scale supersymmetry breaking mass. This is because the vector superfield is not localized in the AdS space, and therefore directly couples to the TeV-brane, which is the source of the supersymmetry breaking. Using (30), the higher Kaluza-Klein modes are approximately given by $m_{n} \simeq(n+1 / 4) \pi k e^{-\pi k R}$. These masses are obtained at tree-level and we will see that interactions of boundary fields with the bulk gauge bosons will generate boundary masses at one-loop. Since the mediation of the supersymmetry breaking is due to gauge interactions, the flavor problem is naturally solved. It is important to note that the theory has a $\mathrm{U}(1)_{R}$ symmetry, since the induced masses are of the Dirac-type instead of the Majorana-type. This is a unique property of these theories, and is due to the $N=2$ bulk supersymmetry.

It is also possible to add hypermultiplets in the bulk, where the fermions have twisted boundary conditions. In particular, if $c=1 / 2$ then the hypermultiplet is conformal, and the resulting Kaluza-Klein spectrum is identical to the vector supermultiplet case.

\subsection{Radiative corrections on the Planck brane}

In order to compute the radiative corrections of the matter fields completely confined on the Planck brane, let us consider the $5 \mathrm{~d}$ AdS propagator for the gauge boson and gaugino. The general expression for the $5 \mathrm{~d}$ propagator in a slice of AdS is derived in the Appendix. Using the expression for the vector field Green's function restricted to the Planck brane $\left(z=z^{\prime}=1 / k\right)$, 
we have

$$
G_{V}\left(x, \frac{1}{k} ; x^{\prime}, \frac{1}{k}\right)=\int \frac{d^{4} p}{(2 \pi)^{4}} e^{i p \cdot\left(x-x^{\prime}\right)} \frac{1}{i p}\left(\frac{J_{0}\left(i p e^{\pi k R} / k\right) Y_{1}(i p / k)-Y_{0}\left(i p e^{\pi k R} / k\right) J_{1}(i p / k)}{J_{0}\left(i p e^{\pi k R} / k\right) Y_{0}(i p / k)-Y_{0}\left(i p e^{\pi k R} / k\right) J_{0}(i p / k)}\right) .
$$

In the limit that $p \ll k e^{-\pi k R}$ we obtain

$$
G_{V}\left(x, \frac{1}{k} ; x^{\prime}, \frac{1}{k}\right) \simeq \frac{-1}{\pi R} \int \frac{d^{4} p}{(2 \pi)^{4}} e^{i p \cdot\left(x-x^{\prime}\right)} \frac{1}{p^{2}}
$$

which reduces to the usual massless vector field Green's function in flat space. In particular notice that by Eq. (35), the charge screening [07, 31] is absent in the slice of AdS since there are no continuum Kaluza-Klein modes. Similarly on the Planck-brane, the twisted gaugino Green's function defined in the Appendix reduces to the form

$$
G_{F}\left(x, \frac{1}{k} ; x^{\prime}, \frac{1}{k}\right)=\int \frac{d^{4} p}{(2 \pi)^{4}} e^{i p \cdot\left(x-x^{\prime}\right)} \frac{1}{i p}\left(\frac{J_{1}\left(i p e^{\pi k R} / k\right) Y_{1}(i p / k)-Y_{1}\left(i p e^{\pi k R} / k\right) J_{1}(i p / k)}{J_{1}\left(i p e^{\pi k R} / k\right) Y_{0}(i p / k)-Y_{1}\left(i p e^{\pi k R} / k\right) J_{0}(i p / k)}\right) .
$$

In the limit that $p \ll k e^{-\pi k R}$ and $k R \gg 1$ the twisted gaugino Green's function becomes

$$
G_{F}\left(x, \frac{1}{k} ; x^{\prime}, \frac{1}{k}\right) \simeq \frac{-1}{\pi R} \int \frac{d^{4} p}{(2 \pi)^{4}} e^{i p \cdot\left(x-x^{\prime}\right)} \frac{1}{p^{2}-\frac{2}{\pi k R}\left(k e^{-\pi k R}\right)^{2}},
$$

which reduces to a massive gaugino Green's function where the gaugino mass agrees with (33). This difference between the gauge boson and gaugino Green's function represents the source of supersymmetry breaking on the Planck brane.

Note that the vector supermultiplet in the bulk, is also equivalent to a conformal hypermultiplet $(c=1 / 2)$ in the bulk, where the fermion has twisted boundary conditions. The 5 -dimensional mass-squared of the scalar is $-3 k^{2}+2 k(\delta(y)-\delta(y-\pi R))$, while the mass of the fermion is $\sigma^{\prime} / 2$ [3]. On the Planck brane $\left(z=z^{\prime}=1 / k\right)$ the twisted fermion Green's function is the same as Eq. (36), while the scalar field Green's function is identical to Eq. (34). In particular, we will also consider the bulk Higgs fields to be conformal hypermultiplets.

The scalar and twisted fermion Green's function on the Planck brane can be used to calculate the one-loop contribution to the mass-squared of boundary matter fields. The boundary matter fields couple to the vector supermultiplet in the bulk via gauge interactions. The Feynman diagrams for the one-loop mass contributions to the boundary scalar fields are the same as those in flat space and can be found in Ref. 225. They give

$$
m_{i}^{2}=4 g^{2} C\left(R_{i}\right) \Pi(0),
$$

where the $4 \mathrm{~d}$ gauge coupling is given by $g^{2}=g_{5}^{2} /(\pi R)$ and we have defined

$$
\Pi(0)=-\pi R \int \frac{d^{4} p}{(2 \pi)^{4}}\left[G_{p}^{(V)}-G_{p}^{(F)}\right] .
$$


The coefficient $C\left(R_{i}\right)$ is the quadratic Casimir of the representation $R_{i}$ in the corresponding gauge group, and $G_{p}$ is the 4 d Fourier transform of the Green's function (see Appendix). Similarly, the boundary matter fields can also couple to a chiral supermultiplet in the bulk 25]. For a conformal supermultiplet $(c=1 / 2)$, where the fermions have twisted boundary conditions we obtain

$$
m_{i}^{2}=Y^{2} \Pi(0)
$$

where $Y$ is the boundary-bulk Yukawa coupling. Assuming that $k=M_{P}$ and $k e^{-\pi k R}=\mathrm{TeV}$ then we obtain $\pi k R=34.54$, and the integral in Eq. (39) can be numerically evaluated to give

$$
\Pi(0)=\frac{0.2525}{2 \pi^{4}}(\mathrm{TeV})^{2} \simeq(0.0360 \mathrm{TeV})^{2} .
$$

This result is finite and insensitive to the ultraviolet cut-off for the same reason that we already explained in the previous section, namely that the supersymmetry breaking is localized on the $\mathrm{TeV}$-brane. In fact, the integration region $p \leq k e^{-\pi k R}=\mathrm{TeV}$ already contributes approximately $90 \%$ of the integral in Eq. (39).

Comparing the above result with the flat space case where 25] $\Pi(0)=\left(0.0367 / R_{\text {flat }}\right)^{2}$, we

see that the two cases are almost numerically identical for a flat-space radius of $R_{\text {flat }}=k e^{-\pi k R}$.

\subsubsection{Superparticle spectrum}

It is now straightforward to extend the above result to the case of the warped MSSM. Assuming that the squarks and sleptons live on the Planck-brane they will receive a one-loop contribution from the bulk gauge and Higgs sector (if they are in the bulk). Assuming the bulk Higgs to be a conformal supermultiplet and following Ref. 25] we obtain

$$
\begin{aligned}
m_{\widetilde{Q}}^{2} & =\left(\frac{4}{3} \alpha_{3}+\frac{3}{4} \alpha_{2}+\frac{1}{60} \alpha_{1}\right) \Pi(0)+\frac{1}{2}\left(\alpha_{t}+\alpha_{b}\right) \Pi(0) \\
m_{\widetilde{U}}^{2} & =\left(\frac{4}{3} \alpha_{3}+\frac{4}{15} \alpha_{1}\right) \Pi(0)+\alpha_{t} \Pi(0) \\
m_{\widetilde{D}}^{2} & =\left(\frac{4}{3} \alpha_{3}+\frac{1}{15} \alpha_{1}\right) \Pi(0)+\alpha_{b} \Pi(0) \\
m_{\widetilde{L}}^{2} & =\left(\frac{3}{4} \alpha_{2}+\frac{3}{20} \alpha_{1}\right) \Pi(0)+\alpha_{\tau} \Pi(0), \\
m_{\widetilde{E}}^{2} & =\frac{3}{5} \alpha_{1} \Pi(0)
\end{aligned}
$$

where the bulk gauge contribution is proportional to the gauge couplings $\alpha_{1,2,3}$ and the conformal bulk Higgs contribution is proportional to the Yukawa couplings $\alpha_{t, b, \tau}$. Thus to obtain an experimentally allowed soft mass spectrum the scale on the $\mathrm{TeV}$ brane should be at least a few $\mathrm{TeV}$. Notice that the dominant corrections are proportional to the gauge couplings. Thus, the lightest scalar field is the right-handed slepton. 


\subsection{Radiative corrections in the slice of AdS}

Consider a conformal hypermultiplet in the bulk with twisted boundary conditions for the fermion. The massless scalar mode $\phi$ in the hypermultiplet will receive a one-loop mass contribution due to the breaking of supersymmetry from fields in the bulk with the twisted boundary conditions. In particular the scalar can couple to the bulk vector supermultiplet. This radiative correction can simply be calculated using the 5-dimensional AdS scalar propagator. For an alternative method to calculate quantum effects in the AdS slice see Ref. [32]. Since the scalar propagates in the bulk we need to integrate over the extra dimension, and the corresponding mass correction is proportional to

$$
\Pi(0)=-\pi R \int \frac{d^{4} p}{(2 \pi)^{4}} \int_{0}^{\pi R} d y\left[G_{p}^{(V)}(z, z)-G_{p}^{(F)}(z, z)\right] \simeq \frac{\Lambda k e^{-2 \pi k R}}{16 \pi^{2}}
$$

where $\Lambda$ is a Planck-scale cutoff. Unlike the radiative corrections of the boundary fields, it turns out that the radiative correction (47) is not finite, as expected from the arguments of the previous section. In fact, the bulk radiative corrections (47) are linearly divergent. This reflects the fact that the bulk fields are propagating in five dimensions and are sensitive to physics on the TeV-brane represented by the cutoff scale $\Lambda e^{-\pi k R}$. This behavior is related to the fact that the supersymmetry breaking mechanism is localized on the TeV-brane and is sensitive to the UV physics. This is different from flat space where the breaking of supersymmetry is inherently a global effect, and consequently the nonlocality produces a finite result.

Let us finally comment on other possible alternatives. If the Higgs is also confined on the Planck-brane, then its mass will be generated at the one-loop level, with a magnitude similar to that of the sleptons (without the Yukawa coupling contribution). Although this contribution will be positive, there are sizeable two-loop effects arising from the squarks that can make the mass-squared negative 25]. Unfortunately, the Higgsino mass cannot be generated by radiative corrections and we will need to extend the model to include a Higgs singlet whose VEV must induce the Higgsino mass. In the above cases we have restricted the observable sector to the Planck-brane. Nevertheless, many more possibilities exist by placing part of the matter in the bulk or on the TeV-brane. For example, consider delocalizing the first two families off the Planck-brane by changing their bulk mass parameters [3]. In this case the corresponding squarks and sleptons of the first two families will have masses larger that those of the third family, a scenario whose phenomenology can have interesting consequences.

\subsection{Relation to $4 \mathrm{~d}$ strongly-coupled CFT}

The AdS/CFT correspondence relates the 5d theory of gravity in AdS to a 4d strongly coupled conformal field theory (CFT) [10]. In the case of a slice of AdS, a similar correspondence can also be formulated [11, 12, 13, 14. The Planck-brane in $\mathrm{AdS}_{5}$ corresponds to an ultraviolet cutoff of the $4 \mathrm{~d}$ CFT and to the gauging of certain global symmetries. For example, in the case we are considering where gravity and the standard model gauge bosons live in the bulk, the corresponding CFT will have the superPoincare group gauged (giving rise to gravity) and 
also the standard model group $\mathrm{SU}(3) \times \mathrm{SU}(2)_{L} \times \mathrm{U}(1)_{Y}$ (giving rise to the standard model gauge bosons and gauginos). Matter on the Planck-brane corresponds to adding new fields to the CFT which only couple to CFT states via gravity and gauge interactions. On the other hand, the TeV-brane corresponds in the dual theory to a infrared cutoff of the CFT [13], 14]. In other words, it corresponds to breaking the conformal symmetry at the TeV-scale. The Kaluza-Klein states of the $5 \mathrm{~d}$ theory correspond to the bound states of the strongly coupled CFT.

This alternate dual description suggests that the supersymmetry-breaking mechanism that we have discussed represents a class of strongly coupled CFT's where supersymmetry is broken at the $\mathrm{TeV}$ scale. The bound states therefore do not respect supersymmetry and give rise to a fermion-boson mass splitting. The 5d warped MSSM is then simply the ordinary 4d MSSM with a strongly coupled CFT sector responsible for the breaking of supersymmetry. The standard model fields coupled to the CFT sector will get tree-level masses while those coupled only via gravity or gauge interactions will receive masses at the one-loop level. In our model the CFT sector is charged under the standard model gauge group and consequently it implies that the gauginos get masses at tree-level. Notice that as we mentioned earlier the gaugino mass is of the Dirac-type. This means that the gaugino has married a fermion bound-state to become massive 1. Since the gaugino mass comes from the mixing between the gaugino and the CFT bound-state, the mass will be proportional to $\sqrt{g^{2} b_{C F T} /\left(8 \pi^{2}\right)}=1 / \sqrt{\pi k R}$, where we have used the AdS/CFT relation [13] $g_{5}^{2} k=8 \pi^{2} / b_{C F T}$ and $g^{2}=g_{5}^{2} /(\pi R)$. This agrees with Eq. (33). Similarly, the smallness of the gravitino mass (of order $10^{-3} \mathrm{eV}$ ) is also easy to understand in the CFT picture. The gravitino coupling to the CFT sector is suppressed by $1 / M_{P}$, so its mass will be of order $\mathrm{TeV}^{2} / M_{P} \sim 10^{-3} \mathrm{eV}$.

Although the CFT picture is useful for understanding some qualitative aspects of the theory, it is practically useless for obtaining quantitative predictions since the theory is strongly coupled. In this sense, the $5 \mathrm{~d}$ gravitational theory in a slice of AdS represents a very useful tool since it allows one to calculate the particle spectrum, which would otherwise be unknown from the CFT side.

\section{Conclusion}

In this paper we have presented a supersymmetric $5 \mathrm{~d}$ theory in warped space where supersymmetry is spontaneously broken by imposing different boundary conditions between the fermion and bosons. While this is reminiscent of the Scherk-Schwarz mechanism in flat space, we have argued that in a warped space this is a novel way of breaking supersymmetry. Unlike the flat-space case where the supersymmetry-breaking mechanism is a global effect, the twisted boundary conditions in the warped space lead to a local supersymmetry breaking effect on the TeV-brane.

A particularly interesting model is the warped MSSM, where matter is confined on the

\footnotetext{
${ }^{4} \mathrm{~A}$ Majorana-type mass would correspond, for example, to a breaking of supersymmetry (in the $5 \mathrm{~d}$ dual) by a nonzero $F_{T}$.
} 
Planck brane, and gravity and gauge fields propagate in the 5d bulk. The gravitino and gaugino receive tree-level masses from the twisted boundary conditions. In particular, the treelevel mass of the gravitino is $\sim 10^{-3} \mathrm{eV}$ and the gaugino mass $\sim \mathrm{TeV}$. The one-loop radiative corrections to the squarks and sleptons confined to the Planck brane are finite and insensitive to the UV cutoff. This simply reflects the fact that the supersymmetry-breaking is localized on the TeV-brane, at a finite distance away from the Planck-brane. The one-loop radiative corrections from the bulk gauge fields are proportional to the gauge couplings and thus naturally solve the flavor problem. If the Higgs sector is also included in the bulk, then the one-loop radiative corrections also give a contribution proportional to the Yukawa couplings. However, in this case the radiative corrections to the Higgs soft mass are not finite. This is in contrast to the flat-space case, and is due to the fact that the bulk Higgs directly couples to the supersymmetry breaking effects on the TeV-brane.

By the AdS/CFT correspondence, the warped supersymmetric standard model can be interpreted in terms of a strongly coupled CFT, where supersymmetry (and conformal symmetry) are broken at the TeV-scale. Thus, the warped MSSM is simply the ordinary 4d MSSM with a strongly coupled CFT which is responsible for breaking supersymmetry. The fact that there exists a weakly coupled $5 \mathrm{~d}$ gravity dual, allows us to calculate the mass spectrum. This provides a powerful tool in obtaining information about the dynamics of this class of strongly coupled CFT's, and is worthy of further investigation.

\section{Acknowledgements}

We wish to thank Emilian Dudas and Dan Waldram for helpful discussions. One of us (TG) acknowledges the Aspen Center for Physics where part of this work was done. The work of TG is supported by the FNRS, contract no. 21-55560.98, while that of AP is partially supported by the CICYT Research Project AEN99-0766.

\section{Appendix: 5D propagators in a slice of AdS}

Let us consider the propagation of bulk fields in a slice of AdS. We will follow the derivation of the Green's function presented in Ref. [33, 12], except that we will extend the previous results to the case of arbitrary bulk fields in the two-brane scenario. The result for the bulk scalar has also recently been given in Ref. [34].

As shown in Ref. [3] the equation of motion for bulk fields $\Phi=\left\{V_{\mu}, \phi, e^{-2 \sigma} \psi_{L, R}\right\}$, can be conveniently written as a second-order differential equation. Thus, introducing a source function $\mathcal{J}$, one obtains

$$
\left[e^{2 \sigma} \eta^{\mu \nu} \partial_{\mu} \partial_{\nu}+e^{s \sigma} \partial_{5}\left(e^{-s \sigma} \partial_{5}\right)-M_{\Phi}^{2}\right] \Phi(x, y)=\mathcal{J}(x, y)
$$

where the parameter $s=\{2,4,1\}$, and the 5 d masses are [3] $M_{\Phi}^{2}=\left\{0, a k^{2}+b \sigma^{\prime \prime}, c(c \pm 1) k^{2} \mp c \sigma^{\prime \prime}\right\}$. 
The corresponding Green's function for (48) can then be defined as

$$
\Phi(x, y)=\int d^{4} x^{\prime} d y^{\prime} \sqrt{-g} G\left(x, y ; x^{\prime}, y^{\prime}\right) e^{(4-s) k y^{\prime}} \mathcal{J}\left(x^{\prime}, y^{\prime}\right) .
$$

provided that $\mathcal{J}=\left\{J_{\mu}, J_{\phi}, \gamma^{\mu} \partial_{\mu} J_{R, L} \pm \partial_{5} J_{L, R}-(c \pm 1) \sigma^{\prime} J_{L, R}\right\}$, where $J_{\mu}, J_{\phi}$ and $J_{L, R}$ are the source terms for the bulk vector, scalar, and fermion, respectively. It is convenient to introduce the variable $z=e^{k y} / k$. In these coordinates the Planck-brane is located at $z^{*}=1 / k$ and the $\mathrm{TeV}$-brane at $z^{*}=e^{\pi k R} / k$. If we now take the $4 \mathrm{~d}$ Fourier transform of the Green's function

$$
G\left(x, z ; x^{\prime}, z^{\prime}\right)=\int \frac{d^{4} p}{(2 \pi)^{4}} e^{i p \cdot\left(x-x^{\prime}\right)} G_{p}\left(z, z^{\prime}\right),
$$

then the Fourier component $G_{p}\left(z, z^{\prime}\right)$ must satisfy the equation

$$
\left(\partial_{z}^{2}+\frac{1-s}{z} \partial_{z}-p^{2}-\frac{\widehat{M}_{\Phi}^{2}}{(k z)^{2}}\right) G_{p}\left(z, z^{\prime}\right)=(k z)^{s-1} \delta\left(z-z^{\prime}\right),
$$

where $\widehat{M}_{\Phi}^{2}=\left\{0, a k^{2}, c(c \pm 1) k^{2}\right\}$. If we define $G_{p}\left(z, z^{\prime}\right)=\left(k^{2} z z^{\prime}\right)^{s / 2} \widehat{G}_{p}\left(z, z^{\prime}\right)$, then Eq. (51) simply becomes the Bessel equation

$$
\left(\partial_{z}^{2}+\frac{1}{z} \partial_{z}-p^{2}-\frac{\alpha^{2}}{z^{2}}\right) \widehat{G}_{p}\left(z, z^{\prime}\right)=(k z)^{-1} \delta\left(z-z^{\prime}\right),
$$

where $\alpha=\sqrt{(s / 2)^{2}+\widehat{M}_{\Phi}^{2} / k^{2}}$. The standard procedure for solving Eq. (52) is to use the solution to the homogeneous equation in the regions $z<z^{\prime}$ and $z>z^{\prime}$, and then impose matching conditions at $z=z^{\prime}$. Thus writing

$$
\widehat{G}_{p}\left(z, z^{\prime}\right)=\theta\left(z-z^{\prime}\right) \widehat{G}_{>}+\theta\left(z^{\prime}-z\right) \widehat{G}_{<}
$$

the solution to the homogeneous equation for $z<z^{\prime}$ is given by

$$
\widehat{G}_{<}\left(z, z^{\prime}\right)=i A_{<}\left(z^{\prime}\right)\left[\widetilde{J}_{\alpha}(i p / k) H_{\alpha}^{(1)}(i p z)-\widetilde{H}_{\alpha}^{(1)}(i p / k) J_{\alpha}(i p z)\right],
$$

and for $z>z^{\prime}$ we obtain

$$
\widehat{G}_{>}\left(z, z^{\prime}\right)=i A_{>}\left(z^{\prime}\right)\left[\widetilde{J}_{\alpha}\left(i p e^{\pi k R} / k\right) H_{\alpha}^{(1)}(i p z)-\widetilde{H}_{\alpha}^{(1)}\left(i p e^{\pi k R} / k\right) J_{\alpha}(i p z)\right],
$$

where $H_{\alpha}^{(1)}=J_{\alpha}+i Y_{\alpha}$ is the Hankel function of the 1st kind of order $\alpha$, and $J_{\alpha}, Y_{\alpha}$ are Bessel functions. If the boundary condition for the Green's function, $\widehat{G}_{<}\left(\widehat{G}_{>}\right)$is even on the Planckbrane (TeV-brane) then [3]

$$
\widetilde{J}_{\alpha}(z)=(-r+s / 2) J_{\alpha}(z)+z J_{\alpha}^{\prime}(z),
$$

where the parameter $r=\{0, b, \mp c\}$, while if the boundary condition is odd then [3]

$$
\widetilde{J}_{\alpha}(z)=J_{\alpha}(z)
$$


and similarly for $\widetilde{H}_{\alpha}^{(1)}$. Note that in the presence of the boundary mass terms parametrized by $r$, the even boundary condition is equivalent to imposing the modified Neumann condition, $\left.\left(\partial_{z}-r \sigma^{\prime}\right) G_{p}\left(z, z^{\prime}\right)\right|_{z=z^{*}}=0$, while the odd boundary condition is equivalent to imposing the Dirichlet condition, $\left.G_{p}\left(z, z^{\prime}\right)\right|_{z=z^{*}}=0$.

The unknown functions $A_{<}\left(z^{\prime}\right)$ and $A_{>}\left(z^{\prime}\right)$ are determined by imposing matching conditions at $z=z^{\prime}$. Continuity of $\widehat{G}_{p}$ at $z=z^{\prime}$ leads to the condition

$$
\left.\widehat{G}_{>}\right|_{z=z^{\prime}}=\left.\widehat{G}_{<}\right|_{z=z^{\prime}},
$$

while the discontinuity in $\partial_{z} \widehat{G}_{p}$ gives the condition

$$
\left.\left(\partial_{z} \widehat{G}_{>}-\partial_{z} \widehat{G}_{<}\right)\right|_{z=z^{\prime}}=\frac{1}{k z^{\prime}} .
$$

This leads to the solutions

$$
\begin{aligned}
& A_{<}\left(z^{\prime}\right)=\frac{\pi}{2 k} \frac{\widetilde{J}_{\alpha}\left(i p e^{\pi k R} / k\right) H_{\alpha}^{(1)}\left(i p z^{\prime}\right)-\widetilde{H}_{\alpha}^{(1)}\left(i p e^{\pi k R} / k\right) J_{\alpha}\left(i p z^{\prime}\right)}{\widetilde{J}_{\alpha}\left(i p e^{\pi k R} / k\right) \widetilde{H}_{\alpha}^{(1)}(i p / k)-\widetilde{H}_{\alpha}^{(1)}\left(i p e^{\pi k R} / k\right) \widetilde{J}_{\alpha}(i p / k)} \\
& A_{>}\left(z^{\prime}\right)=\frac{\pi}{2 k} \frac{\widetilde{J}_{\alpha}(i p / k) H_{\alpha}^{(1)}\left(i p z^{\prime}\right)-\widetilde{H}_{\alpha}^{(1)}(i p / k) J_{\alpha}\left(i p z^{\prime}\right)}{\widetilde{J}_{\alpha}\left(i p e^{\pi k R} / k\right) \widetilde{H}_{\alpha}^{(1)}(i p / k)-\widetilde{H}_{\alpha}^{(1)}\left(i p e^{\pi k R} / k\right) \widetilde{J}_{\alpha}(i p / k)} .
\end{aligned}
$$

Finally substituting these functions into the equations for $\widehat{G}_{>}$and $\widehat{G}_{<}$gives the expression for the Green's function in a slice of AdS

$$
\begin{aligned}
G_{p}\left(z, z^{\prime}\right)= & i \frac{\pi}{2} k^{s-1}\left(z z^{\prime}\right)^{s / 2}\left[\frac{\widetilde{J}_{\alpha}\left(i p e^{\pi k R} / k\right) H_{\alpha}^{(1)}\left(i p z_{>}\right)-\widetilde{H}_{\alpha}^{(1)}\left(i p e^{\pi k R} / k\right) J_{\alpha}\left(i p z_{>}\right)}{\widetilde{J}_{\alpha}\left(i p e^{\pi k R} / k\right) \widetilde{H}_{\alpha}^{(1)}(i p / k)-\widetilde{H}_{\alpha}^{(1)}\left(i p e^{\pi k R} / k\right) \widetilde{J}_{\alpha}(i p / k)}\right] \\
& \times\left[\widetilde{J}_{\alpha}(i p / k) H_{\alpha}^{(1)}\left(i p z_{<}\right)-\widetilde{H}_{\alpha}^{(1)}(i p / k) J_{\alpha}\left(i p z_{<}\right)\right],
\end{aligned}
$$

where we have defined $z_{>}\left(z_{<}\right)$to be the greater (lesser) of $z$ and $z^{\prime}$. The Green's function (62) is the general expression for arbitrary bulk fields in a slice of AdS.

The Kaluza-Klein mass spectrum can be obtained from the pole condition of the Green's function, namely

$$
\widetilde{J}_{\alpha}\left(i p e^{\pi k R} / k\right) \widetilde{H}_{\alpha}^{(1)}(i p / k)-\widetilde{H}_{\alpha}^{(1)}\left(i p e^{\pi k R} / k\right) \widetilde{J}_{\alpha}(i p / k)=0
$$

This leads to the condition

$$
\frac{\widetilde{J}_{\alpha}\left(\frac{m}{k}\right)}{\widetilde{Y}_{\alpha}\left(\frac{m}{k}\right)}=\frac{\widetilde{J}_{\alpha}\left(\frac{m}{k} e^{\pi k R}\right)}{\widetilde{Y}_{\alpha}\left(\frac{m}{k} e^{\pi k R}\right)},
$$

where the four-momentum $p^{2}=-m^{2}$. The solutions of this equation for the various combinations of boundary conditions on the Planck and TeV-branes, reproduces all the Kaluza-Klein mass spectrum results from [3]. 
For the twisted boundary condition, $\Phi(0)=\Phi(0)$ and $\Phi(\pi R)=-\Phi(\pi R)$, we must impose on the corresponding Green's function the even boundary condition on the Planck-brane, Eq. (56), and the odd boundary condition on the TeV-brane, Eq. (57). The mass spectrum is obtained by solving (64) which now becomes

$$
\frac{(-r+s / 2) J_{\alpha}\left(\frac{m}{k}\right)+\frac{m}{k} J_{\alpha}^{\prime}\left(\frac{m}{k}\right)}{(-r+s / 2) Y_{\alpha}\left(\frac{m}{k}\right)+\frac{m}{k} Y_{\alpha}^{\prime}\left(\frac{m}{k}\right)}=\frac{J_{\alpha}\left(\frac{m}{k} e^{\pi k R}\right)}{Y_{\alpha}\left(\frac{m}{k} e^{\pi k R}\right)},
$$

and for the fermion and gravitino the equation simplifies further to Eq. (29).

\section{References}

[1] N. Arkani-Hamed, S. Dimopoulos and G. Dvali, "The hierarchy problem and new dimensions at a millimeter," Phys. Lett. B429 (1998) 263 [hep-ph/9803315.

[2] L. Randall and R. Sundrum, "A large mass hierarchy from a small extra dimension," Phys. Rev. Lett. 83 (1999) 3370 [hep-ph/9905221].

[3] T. Gherghetta and A. Pomarol, "Bulk fields and supersymmetry in a slice of AdS," Nucl. Phys. B586 (2000) 141 hep-ph/0003129.

[4] R. Altendorfer, J. Bagger and D. Nemeschansky, "Supersymmetric Randall-Sundrum scenario," hep-th/0003117.

[5] N. Alonso-Alberca, P. Meessen and T. Ortin, "Supersymmetric brane-worlds," Phys. Lett. B482 (2000) 400 hep-th/0003248; A. Falkowski, Z. Lalak and S. Pokorski, "Supersymmetrizing branes with bulk in five-dimensional supergravity," Phys. Lett. B491 (2000) 172 [hep-th/0004093; E. Bergshoeff, R. Kallosh and A. Van Proeyen, "Supersymmetry in singular spaces," JHEP0010 (2000) 033 [hep-th/0007044].

[6] See for example, H. Verlinde, "Holography and compactification," Nucl. Phys. B580 (2000) 264 [hep-th/9906182]; M. J. Duff, J. T. Liu and K. S. Stelle, "A supersymmetric type IIB Randall-Sundrum realization," hep-th/0007120; M. Cvetic, M. J. Duff, J. T. Liu, H. Lu, C. N. Pope and K. S. Stelle, "Randall-Sundrum brane tensions," hep-th/0011167.

[7] A. Pomarol, "Grand unified theories without the desert," Phys. Rev. Lett. 85 (2000) 4004 hep-ph/0005293].

[8] I. Antoniadis, “A Possible New Dimension At A Few Tev," Phys. Lett. B246 (1990) 377.

[9] A. Pomarol, "Gauge bosons in a five-dimensional theory with localized gravity," Phys. Lett. B486 (2000) 153 hep-ph/9911294.

[10] J. Maldacena, "The large $N$ limit of superconformal field theories and supergravity," Adv. Theor. Math. Phys. 2 (1998) 231 hep-th/9711200. 
[11] S. S. Gubser, "AdS/CFT and gravity," hep-th/9912001; M. J. Duff and J. T. Liu, "On the equivalence of the Maldacena and Randall-Sundrum pictures," Phys. Rev. Lett. 85 (2000) 2052 hep-th/0003237.

[12] S. B. Giddings, E. Katz and L. Randall, "Linearized gravity in brane backgrounds," JHEP0003 (2000) 023 hep-th/0002091.

[13] N. Arkani-Hamed, M. Porrati and L. Randall, "Holography and Phenomenology," hep-th/0012148.

[14] R. Rattazzi and A. Zaffaroni, "Comments on the Holographic Picture of the RandallSundrum Model," hep-th/0012248.

[15] I. Oda, "Localization of gravitino on a brane," hep-th/0008134; A. Falkowski, Z. Lalak and S. Pokorski, "Five-dimensional gauged supergravities with universal hypermultiplet and warped brane worlds," hep-th/0009167.

[16] W. D. Goldberger and M. B. Wise, "Bulk fields in the Randall-Sundrum compactification scenario," Phys. Rev. D60 (1999) 107505 hep-ph/9907218.

[17] H. Davoudiasl, J. L. Hewett and T. G. Rizzo, "Bulk gauge fields in the Randall-Sundrum model," Phys. Lett. B473 (2000) 43 hep-ph/9911262].

[18] B. Bajc and G. Gabadadze, "Localization of matter and cosmological constant on a brane in anti de Sitter space," Phys. Lett. B474 (2000) 282 [hep-th/9912232]; Y. Grossman and M. Neubert, "Neutrino masses and mixings in non-factorizable geometry," Phys. Lett. B474 (2000) 361 hep-ph/9912408; S. Chang, J. Hisano, H. Nakano, N. Okada and M. Yamaguchi, "Bulk standard model in the Randall-Sundrum background," Phys. Rev. D 62 (2000) 084025 hep-ph/9912498.

[19] M. A. Luty and R. Sundrum, "Hierarchy Stabilization in Warped Supersymmetry," hep-th/0012158; J. Bagger, D. Nemeschansky and R. Zhang, "Supersymmetric Radion in the Randall-Sundrum Scenario," hep-th/0012163.

[20] For a review, see H. P. Nilles, "On the low-energy limit of string and M-theory," hep-ph/0004064.

[21] I. Brevik, K. A. Milsimilarton, S. Nojiri and S. D. Odintsov, "Quantum (in)stability of a brane-world AdS(5) universe at nonzero temperature," hep-th/0010205.

[22] J. Scherk and J. H. Schwarz, "Spontaneous Breaking Of Supersymmetry Through Dimensional Reduction," Phys. Lett. B82 (1979) 60; "How To Get Masses From Extra Dimensions," Nucl. Phys. B153 (1979) 61.

[23] I. Antoniadis, C. Munoz and M. Quiros, "Dynamical supersymmetry breaking with a large internal dimension," Nucl. Phys. B397 (1993) 515 [hep-ph/9211309]; I. Antoniadis and K. Benakli, "Limits on extra dimensions in orbifold compactifications of superstrings," 
Phys. Lett. B326 (1994) 69 [hep-th/9310151]; K. Benakli, "Perturbative Supersymmetry Breaking In Orbifolds With Wilson Line Backgrounds," Phys. Lett. B386 (1996) 106 hep-th/9509115.

[24] A. Pomarol and M. Quiros, "The standard model from extra dimensions," Phys. Lett. B438 (1998) 255 hep-ph/9806263.

[25] I. Antoniadis, S. Dimopoulos, A. Pomarol and M. Quiros, "Soft masses in theories with supersymmetry breaking by TeV-compactification," Nucl. Phys. B544 (1999) 503 [hep-ph/9810410]; A. Delgado, A. Pomarol and M. Quiros, "Supersymmetry and electroweak breaking from extra dimensions at the TeV-scale," Phys. Rev. D 60 (1999) 095008 [hep-ph/9812489].

[26] R. Barbieri, L. J. Hall and Y. Nomura, "A constrained standard model from a compact extra dimension," hep-ph/0011311.

[27] E. Dudas and C. Grojean, "Four-dimensional M-theory and supersymmetry breaking," Nucl. Phys. B507 (1997) 553 [hep-th/9704177]; I. Antoniadis and M. Quiros, "Supersymmetry breaking in M-theory and gaugino condensation," Nucl. Phys. B505 (1997) 109 [hep-th/9705037]; A. Lukas, B. A. Ovrut and D. Waldram, "Gaugino condensation in M-theory on $S^{* *} 1 / Z(2)$," Phys. Rev. D 57 (1998) 7529 hep-th/9711197; M. Fabinger and P. Horava, "Casimir effect between world-branes in heterotic M-theory," Nucl. Phys. B580 (2000) 243 hep-th/0002073.

[28] I. Antoniadis, S. Dimopoulos and G. Dvali, "Millimeter range forces in superstring theories with weak-scale compactification," Nucl. Phys. B516 (1998) 70 hep-ph/9710204].

[29] S. Ferrara, C. Kounnas and F. Zwirner, "Mass formulae and natural hierarchy in string effective supergravities," Nucl. Phys. B429 (1994) 589 hep-th/9405188].

[30] T. Gherghetta, "The role of scalar and pseudoscalar fields in determining nucleosynthesis bounds on the scale of supersymmetry breaking," Phys. Lett. B423 (1998) 311 hep-ph/9712343]; A. Brignole, F. Feruglio and F. Zwirner, "Signals of a superlight gravitino at $e+e$-colliders when the other superparticles are heavy," Nucl. Phys. B516 (1998) 13 hep-ph/9711516.

[31] N. Kaloper, E. Silverstein and L. Susskind, "Gauge symmetry and localized gravity in M theory," hep-th/0006192.

[32] J. Garriga, O. Pujolas and T. Tanaka, "Radion effective potential in the brane-world," hep-th/0004109; S. Nojiri, S. D. Odintsov and S. Zerbini, "Bulk versus boundary (gravitational Casimir) effects in quantum creation of inflationary brane world universe," Class. Quant. Grav. 17 (2000) 4855 [hep-th/0006115].

[33] J. Garriga and T. Tanaka, "Gravity in the brane-world," Phys. Rev. Lett. 84 (2000) 2778 hep-th/9911055. 
[34] B. Grinstein, D. R. Nolte and W. Skiba, "On a covariant determination of mass scales in warped backgrounds," hep-th/0012074. 